\title{
The Impact of Structure and Oxygen Vacancies on Lowering the Overpotential
}

\author{
Xueqing Zhang ${ }^{* 1}$, Peter Klaver ${ }^{2}$, Rutger van Santen ${ }^{3}$, M.C.M. van de Sanden ${ }^{1,4}$, Anja \\ Bieberle-Hütter*1 \\ 1 Electrochemical Materials and Interfaces, Dutch Institute for Fundamental Energy Research \\ (DIFFER), Eindhoven, the Netherlands \\ 2 Dutch Institute for Fundamental Energy Research (DIFFER), Partner in the Trilateral Euregio \\ Cluster, Eindhoven, The Netherlands \\ 3 Institute for Complex Molecular Systems (ICMS), Eindhoven University of Technology (TU/e), the \\ Netherlands \\ 4 Plasma and Materials Processing, Department of Applied Physics, Eindhoven University of \\ Technology (TU/e), the Netherlands
}

\begin{abstract}
:
Simulations of the oxygen evolution reaction (OER) are essential for understanding the limitations of water splitting. Most research has focused so far on the OER at flat metal oxide surfaces. The structure sensitivity of the OER has, however, recently been highlighted as a promising research direction. To probe the structure sensitivity, we investigate the OER at eleven hematite (Fe2O3) surfaces with density functional theory + Hubbard U (DFT + U) calculations. The results show that the $\mathrm{O}-\mathrm{O}$ coupling ( $\mathrm{O}-\mathrm{O}$ bond formation via two adjacent terminal $\mathrm{Os}$ at dual site) OER mechanism at the (110) surface is competing with the mechanism of $\mathrm{OOH}$ formation at single site. We study the effects of surface orientation (110 vs. 104), active surface sites (bridge vs. terminal site), presence of surface steps and oxygen vacancy concentration on the OER and explore strategies to reduce the OER overpotential. It is found that the oxygen vacancy concentration is the most effective parameter in reducing the overpotential. In particular, an overpotential of as low as $0.47 \mathrm{~V}$ is obtained for the (110) surface with an oxygen vacancy concentration of 1.26 vacancies $/ \mathrm{nm}^{2}$.
\end{abstract}

Keywords: Oxygen evolution reaction, $\mathrm{DFT}+\mathrm{U}$, Water splitting, Overpotential, Hematite

The design of photoelectrodes for water splitting in photoelectrochemical (PEC) conversion of solar into chemical energy is both fundamentally and practically important. ${ }^{1-10}$ Hematite $\left(\alpha-\mathrm{Fe}_{2} \mathrm{O}_{3}\right)$ has emerged as a promising photoelectrode material and received much attention due to its suitable band gap of about $2.1 \mathrm{eV}$, an excellent chemical stability in a broad $\mathrm{pH}$ range, its natural abundance, nontoxicity and low cost. ${ }^{11-13}$ However, one main drawback of hematite as an efficient photoelectrode material in PEC water splitting is its high oxygen evolution reaction (OER) overpotential. To improve the solar-to-hydrogen conversion, a significant reduction of the overpotential is urgently needed as discussed in a recent review paper by Zhang and Bieberle-Hütter. ${ }^{14}$ In order to reduce the overpotential, in-depth understanding of the key aspects of the OER is required. There are many mechanisms proposed for the OER as discussed in ref. ${ }^{14}$ However, common agreement has not been achieved yet. The interpretation of experimental electrochemical measurements is difficult due to the solid-liquid interface and the non-direct measurement methods for surface species, reaction rates and products. Advances in modeling and simulation 
techniques allow for computational design at an atomistic level. ${ }^{15-23}$

The simulation of photoexcitation as the driving force of the water splitting reaction has only been demonstrated for small model systems. ${ }^{24,25}$ In most of the literature, water decomposition at the semiconductor surface is viewed as an electro-catalytic process driven by the electrochemical potential. Most of the DFT calculations have been done using a solid-gas model. ${ }^{19}$, 26-31 DFT calculations with explicit water molecules have also been performed. ${ }^{32-34}$ Such models are more realistic to simulate the electrode-electrolyte interface, however, they are less common and computationally more expensive. ${ }^{32-34}$ The investigation of the OER under dark conditions with a solid-gas model is the first step and is therefore the main topic of this paper.

Theoretical calculations on this topic have so far merely been focused on the hematite (0001) surface. By using DFT + U calculations, Liao et al. ${ }^{35}$ explored the effects of doping on the water oxidation reaction on the fully hydroxylated hematite (0001) surface. Cation doping was realized by substitution of $\mathrm{Fe}$ by $\mathrm{Ti}, \mathrm{Mn}, \mathrm{Co}$ and $\mathrm{Ni}$; anion doping was realized by replacing $\mathrm{O}$ by $\mathrm{F}$. The reaction energetics on pure and doped hematite surfaces were discussed. The authors found that Co- or Ni-doped hematite surfaces give the most thermodynamically favored reaction pathway. Co or $\mathrm{Ni}$ doping reduces the overpotential up to $0.15 \mathrm{~V}$. In contrast, $\mathrm{Ti}, \mathrm{Mn}, \mathrm{Si}$, and $\mathrm{F}$ doping increased the overpotential beyond that of pure hematite, suggesting $\mathrm{Co}$ and $\mathrm{Ni}$ additions are candidates to improve the catalytic activity of pure hematite. By doing similar calculations, Neufeld and Toroker ${ }^{29}$ found an unfavourable increase in the overpotential for oxidizing water at hematite (0001) surface upon platinum doping. Nguyen et al. ${ }^{31}$ investigated water oxidation on hematite (0001) with vacancies. They found that iron vacancies do not reduce the OER overpotential, whereas oxygen vacancies lower the overpotential by $\sim 0.3 \mathrm{~V}$ compared to the defect free surface. ${ }^{31}$ More recently, Hellman et al. ${ }^{36}$ studied the OER on hydroxyl- and oxygenterminated hematite (0001) surfaces. The authors found that the effect of oxygen vacancies on reducing the overpotential depends on the surface termination.

In summary, significant theoretical contributions have been made to simulate the OER at the hematite (0001) surface. ${ }^{29}, 31,35-46$ However, the hematite thin films in PEC water splitting experiments are dominated by the (110) and the (104) surfaces 47 and surface orientation dependence of the OER is highly feasible. A recent experimental paper nicely illustrated how the photocurrent density can be increased from $0.02 \mathrm{~mA} / \mathrm{cm}^{2}$ to $0.65 \mathrm{~mA} / \mathrm{cm}^{2}$ (both at $1.55 \mathrm{~V}$ vs. reversible hydrogen electrode $(\mathrm{RHE})$ ) for samples fully oriented in (104) and (110) directions, respectively. This strong increase was attributed to different electron and hole mobilities in the different planes as well as to the oxygen terminations. ${ }^{47}$ It should, however, be noted that these results refer to photocurrent measurements under illumination and are not merely attributed to the dark OER overpotentials. The relation of the OER overpotential and surface orientation is, however, of considerable interest and can be a good strategy to increase the performance of photo-electrode materials.

It is desirable to have an atomistic view of the surface orientation/termination dependent OER activities on the theoretical side. In this paper we compare the OER at the experimentally most prominent surfaces of hematite, i.e. (104) and (110), respectively.

Electrocatalytic activity and selectivity is determined by the properties of the catalyst surface. By controlling the atomic structure of the catalyst surface it is possible to provide new strategies to improve the water splitting efficiency. We investigate some possibilities of especially active sites for oxygen evolution for better activity. To explore the best strategies to reduce the OER overpotential at the (110) surface, we study the effects of active sites, presence of surface steps and sublayer oxygen vacancies on the overpotential.

Density functional theory (DFT) calculations have been performed using the ab-initio total-energy and molecular dynamics program VASP (Vienna Ab initio Simulation Package) developed by the Fakultät für 
Physik of the Universitat Wien. ${ }^{48-52}$ Since $\mathrm{Fe}_{2} \mathrm{O}_{3}$ contains highly correlated $3 d$ electrons, we chose the spin polarized $\mathrm{DFT}+\mathrm{U}$ formalism ${ }^{35}$ due to improper treatment of the d-electrons with standard DFT. The $U$ value of $4.3 \mathrm{eV}$ for Fe was derived in the literature ${ }^{35}$ and has been applied to many hematite systems. ${ }^{16,} 29$ The Perdew-Burke-Ernzerhof (PBE) XC functional ${ }^{53}$ and the projected augmented wave (PAW) ${ }^{49}, 54$ potentials were used. Experimental PEC water oxidation is usually operated under high $\mathrm{pH}$ condition, at which the surfaces are deprotonated. Thus, the initial surfaces in this study are oxygen terminated. We use in this study a solid-gas model similarly as in other OER studies of hematite. ${ }^{29}$, 31, 35 More computational details are provided in the supporting information.

Several mechanisms are suggested for the OER from metal oxide surfaces in the literature. ${ }^{14} \mathrm{At}$ present, the most widely assumed OER mechanism is the one proposed by Rossmeisl et al. ${ }^{21}$ This mechanism, focusing merely on the electrochemical steps, consists of four proton-coupled electron transfer steps as shown in Figure 1. It has become very popular and has been shown to predict trends for the OER quite well. ${ }^{14}$, 17, 28, $29,55,56$ The effect of a bias on all states involving an electron in the electrode is included by shifting the energy of this state by $\Delta G_{U}=-e U$, where $U$ is the electrode potential relative to the standard hydrogen electrode. ${ }^{21}$

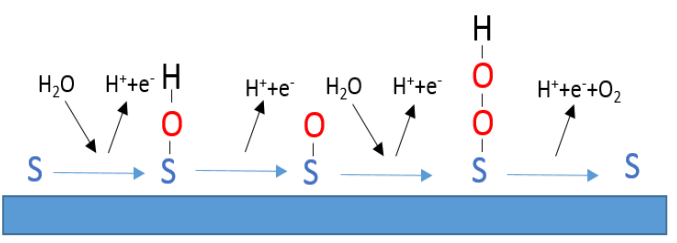

Figure 1. Sketch of the OER mechanism according to Rossmeisl et al. ${ }^{21} ; \mathrm{S}$ is the active surface site.

Figure $2(a)$ and (b) show the schematics of $\mathrm{OH}$ adsorption on two possible adsorption sites on a hematite (110) surface, i.e. the terminal and the bridge site position, respectively. Figure 2 (c-f) illustrate the molecular geometries with intermediate species for the terminal site of the hematite (110) surface. The OER at a bridge site of the (110) surface was also calculated but not shown in this figure. The images of Figure 2 (cf) refer to $\mathrm{S}, \mathrm{S}-\mathrm{O}-\mathrm{H}, \mathrm{S}-\mathrm{O}, \mathrm{S}-\mathrm{O}-\mathrm{O}-\mathrm{H}$ in Figure 1, respectively. The free energies of these four states are calculated to analyse the four steps shown in Figure 1. We use previously reported values for zero point energy (ZPE) correction and entropy contribution ( $\mathrm{T} \Delta \mathrm{S})$, since they were previously found to be very similar between different oxide materials. ${ }^{29,} 35$ Kanan et al. ${ }^{57}$ compared the ZPE corrections of substrates $\mathrm{MnO}: \mathrm{ZnO}, \mathrm{TiO}_{2}$ and $\mathrm{Fe}_{2} \mathrm{O}_{3}$. The authors found that the ZPE corrections calculated for OER reactive species on the $\mathrm{MnO}: \mathrm{ZnO}$ $(001)^{57}$ surface are very close to those of density functional theory generalized gradient approximation (DFT-GGA) calculations for rutile $\mathrm{TiO}_{2}(110)^{19}$ and $\mathrm{GGA}+U$ calculations for $\mathrm{Fe}_{2} \mathrm{O}_{3}(0001)^{35}$, with differences below $0.07 \mathrm{eV}$. (a)

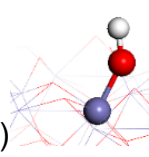

(b)
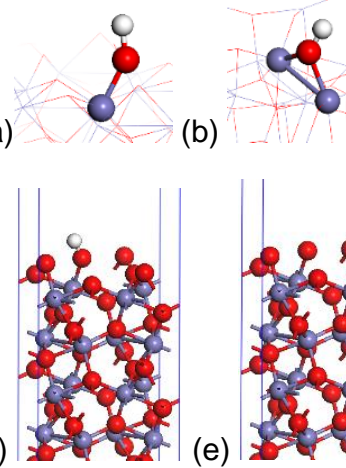

(e) (c)
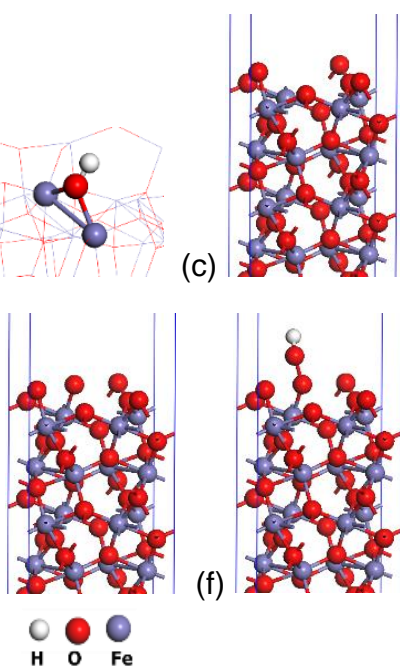

(f)

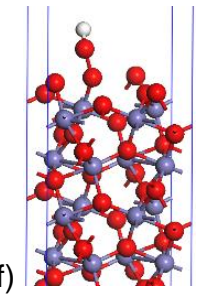

Figure 2. Schematic of $\mathrm{OH}$ adsorption on $\mathrm{Fe}_{2} \mathrm{O}_{3}(110)$ terminal (a) and bridge (b) site with the adsorbate and the active surface site (Fe atom) highlighted for guiding the eyes; side view of the molecular geometry with intermediate species, free site (c), $\mathrm{OH}$ (d), $\mathrm{O}(\mathrm{e})$, and $\mathrm{OOH}(\mathrm{f})$.

According to the method described in ref. ${ }^{21}$ the oxygen evolution reaction (OER) overpotential is determined by

$$
\eta=\max \left[\Delta G_{n}\right] / e-1.23[V]
$$

where $\Delta \mathrm{G}$ signifies the free energy with the free site as the reference. $\mathrm{n}$ is the number of reactions considered in the system and $\Delta G_{n}$ is the free energy step for a single reaction.

Figure 3 (a) and (b) show the free energy for the two active sites (terminal and bridge) for two surface orientations, i.e. (110) and (104), as a function of the reaction step. For the terminal site (Figure 3a), the free 
energy is lower for the (110) orientation than for the (104) orientation for all intermediates. The difference is most pronounced for the formation of $\mathrm{OH}$ and $\mathrm{O}$, respectively. The largest step size $\Delta G$ is found for both orientations for the formation of $\mathrm{O}$; hence, the deprotonation step to form $\mathrm{O}$ is considered to be rate limiting on terminal sites. The OER overpotentials are $1.01 \mathrm{~V}$ and $0.79 \mathrm{~V}$ for the (104) and the (110) surface, respectively.

For the bridge site (Figure $3 b$ ), the situation is different: the free energy for all intermediates is lower for the (104) orientation compared to the (110) orientation. Similar to the terminal site, the formation of $\mathrm{O}$ on the bridge site requires the highest energy and is therefore rate limiting. The overpotentials for this step are $0.92 \mathrm{~V}$ and $0.78 \mathrm{~V}$ for (104) and (110), respectively.
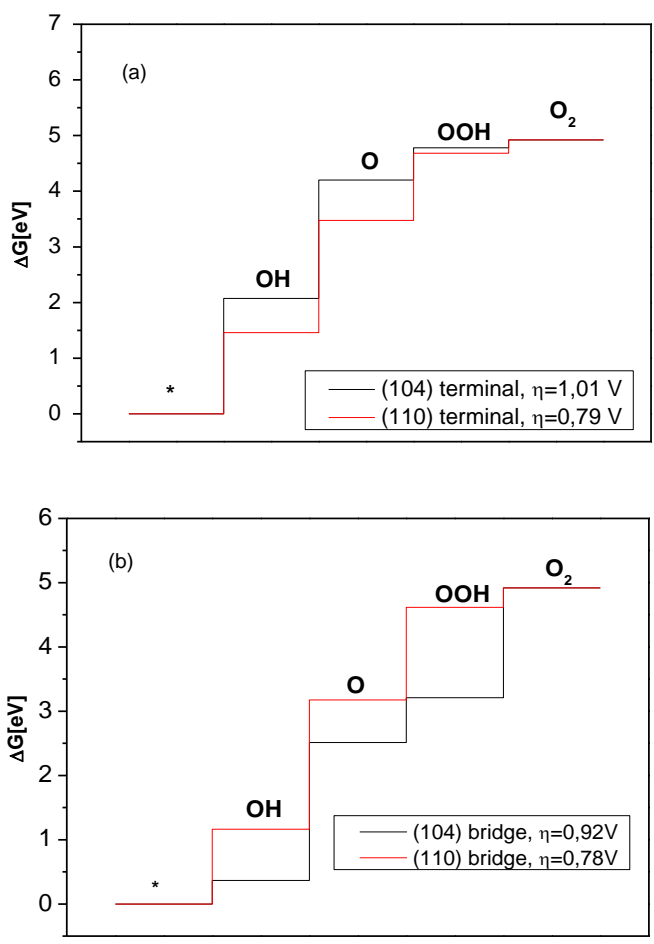

Figure 3. Free energy vs. reaction step for water oxidation on $\mathrm{Fe}_{2} \mathrm{O}_{3}(104)$ and (110) surfaces: a) terminal site, b) bridge site.

In summary, for both terminal and bridge sites, $\mathrm{O}$ formation by deprotonation from $\mathrm{OH}$ is the limiting reaction in this mechanism. Independent of the site, the (110) surface has lower overpotential than the (104) surface and is, hence, more active towards the OER; no significant differences between the overpotentials between terminal and bridge site are found. This trend is in agreement with recent experimental mesurements. ${ }^{47}$ Kment et al. ${ }^{47}$ synthesised thin hematite films exhibiting controlled crystal orientation. The precise control of the synthetic conditions allowed fabricating hematite photo-anodes exhibiting fully textured structures along (110) and (104) crystal planes. Very different photocurrents of $0.65 \mathrm{~mA} / \mathrm{cm}^{2}$ and 0.02 $\mathrm{mA} / \mathrm{cm}^{2}$ (at $1.55 \mathrm{~V}$ vs. RHE) were found for the (110) and the (104) samples, respectively. ${ }^{47}$ There is also a significant difference in onset potential of about $1.05 \mathrm{vs}$. $1.55 \mathrm{~V}$ vs. RHE for (110) and (104), respectively. In our simulations, we also found that the (110) surface is more active than the (104) surface for the OER; however, the differences in the overpotentials are not large, $0.22 \mathrm{~V}$ and $0.14 \mathrm{~V}$ for terminal and bridge site, respectively. This confirms the interpretations of Kment at al. that the large performance differences between (110) and (104) surfaces are more related to difference in photo-absorption and/or charge transport properties 47 than to electrochemical activation.

The OER mechanism that we discussed in 3.1 and 3.2 considers a process where oxygen molecules are formed by an associative mechanism on the anode via a surface $-\mathrm{OOH}$ intermediate. Direct recombination of oxygen atoms to form $\mathrm{O}_{2}$ was excluded in the literature ${ }^{21}$ because a large activation barrier is expected for this process. ${ }^{58}$ However, from our simulations, the $\mathrm{O}-\mathrm{O}$ coupling (the bond formation between two adjacent terminal $O s$ ) at full $O$ termination of the (110) surface is a spontaneous process. Figure 4 shows the different geometries of a fully oxygen terminated hematite (110) surface: in the initial geometry two $\mathrm{O}$ atoms are separately bonded to $\mathrm{Fe}$ atoms; after spontaneous O-O coupling, the final geometry is reached and the two $\mathrm{O}$ atoms are bonded. Such spontaneous O-O formation has also been reported on the hematite (0001) surface by Nguyen et al. ${ }^{38}$ 


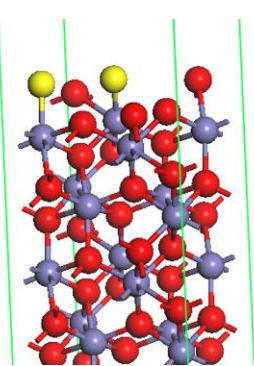

(a)

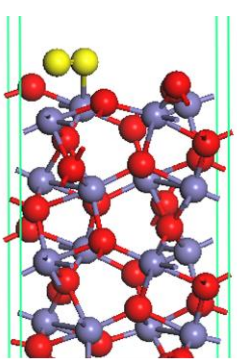

(b)
Figure 4. The initial (a) and final (b) geometry of the fully oxygen terminated hematite (110) surface.

Considering spontaneous $\mathrm{O}-\mathrm{O}$ formation, we explore the OER mechanism of O-O formation via two adjacent terminal $O$ atoms, i.e. the OER occurs at dual sites. This is in contrast to the mechanism used in 3.1 and 3.2. OER mechanisms with two or more sites have been proposed in many studies. ${ }^{12,14,59-63}$ For instance, using in-situ infrared (IR) adsorbed spectroscopy, Nakamura et al. claimed the occurrence of surface $\mathrm{OO}$ and $\mathrm{OOH}$ species. ${ }^{59,60}$ They proposed a mechanism of nucleophilic attack combined with oxidation that is intermediated by holes, followed by the coupling of $-\mathrm{OH}$ groups adsorbed on the surface $\left(\mathrm{TiO}_{2}\right) .{ }^{59,60}$ Recently, Formal and co-workers ${ }^{12}$ presented an experimental study with the first rate law analysis of photo-induced water oxidation on a photoanode surface. Using photoinduced absorption spectroscopy and step on/off photo current measurements, they suggest two possible mechanisms (single site and $\mathrm{O}-\mathrm{O}$ coupling on dual site) of water oxidation on a hematite surface at high $\mathrm{pH} .{ }^{12}$

In general, we can imagine two reaction paths for the O-O coupling. The difference between the two is the sequence of the second water addition (see Figure 5). In mechanism 1 (M1), the second water addition occurs after the first deprotonation. In mechanism 2 (M2), in contrast, the second water addition is before the first deprotonation.

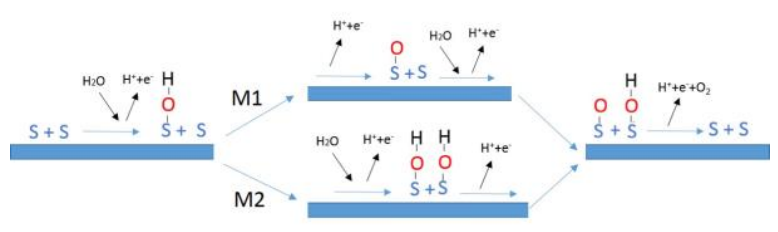

Figure 5. Sketch of OER O-O coupling mechanism 1 (M1, top) and mechanism 2 (M2, bottom).

Figure 6 shows the free energy profile of the two O-O coupling mechanisms M1 and M2 as sketched in Figure 5. All calculated free energies are the same except of the second step which is the formation of $\mathrm{O}$ in $\mathrm{M} 1$ and the formation of $\mathrm{OH}+\mathrm{OH}$ in $\mathrm{M} 2$. In M1, the rate limiting step is the third step (formation of $\mathrm{OH}+\mathrm{O}, \eta=$ $0.85 \mathrm{~V}$ ), whereas it is the second step (formation of $\mathrm{OH}$ $+\mathrm{OH}, \eta=0.79 \mathrm{~V})$ in $\mathrm{M} 2$. Hence, the rate limiting step, i.e. the overpotential, is lower for M2 compared to M1 and it refers two different reactions. The overpotential of dual site M2 $(0.79 \mathrm{~V})$ is the same as for the terminal single site mechanism on the (110) surface, although the rate limiting reactions are different, formation of $\mathrm{OH}$ $+\mathrm{OH}$ and $\mathrm{O}$, for dual site and single site respectively. Therefore, the $\mathrm{O}-\mathrm{O}$ coupling mechanism M2 at hematite (110) surface is also a possible mechanism and is competing with the mechanism of $\mathrm{OOH}$ formation (Figure 1). ${ }^{21}$

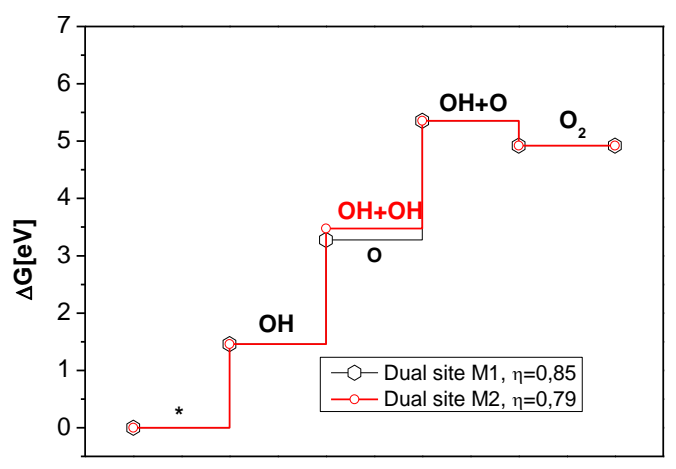

Figure 6. Free energy versus reaction step for the two O-O coupling mechanism on the (110) surface. M1 refers to the mechanism shown in the top part of Figure 5, with the third step being rate limiting. $\mathrm{M} 2$ refers to the mechanism shown in the bottom part of Figure 5, with the second step being rate limiting.

While structure sensitivity, particularly the presence of surface steps, of chemical reactions was found to be very important for metal surfaces, ${ }^{64,65}$ it has 
been investigated significantly less for metal oxides. ${ }^{66}$ It is therefore important to investigate the effects of surface steps on the OER activities. Figure 9 shows a surface step at a hematite (110) surface. The OER at the adsorption sites of the lower (Figure 7a) and upper edge (Figure 7b) are calculated based on the mechanism discussed in chapter 3.1.21 Figure 8 shows the free energy versus reaction step of the OER on the flat surface, the lower step edge, and the upper step edge. Overall, the differences in free energy are rather small. For all geometries, the rate limiting reaction is the formation of $\mathrm{O}$. It is found that the overpotential decreases for the lower step edge $(\eta=0.74 \mathrm{~V})$ compared to the flat surface $(\eta=0.79 \mathrm{~V})$, whereas it increases at the upper step edge $(\eta=0.82 \mathrm{~V})$. This is attributed to destabilization of $\mathrm{OH}$ with respect to $\mathrm{O}$ at the lower step edge, which reduces the limiting free energy step $\left(\Delta G_{2}\right)$. By contrast, at the upper step edge $\mathrm{O}$ is destabilized with respect to $\mathrm{OH}$ which leads to a different situation and results in an increase of the overpotential.

(a)

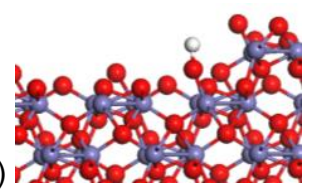

(b)

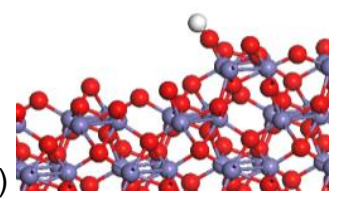

Figure 7. $\mathrm{OH}$ adsorption at step edge of (110) surface: a) adsorption at lower step edge; b) adsorption at upper step edge.

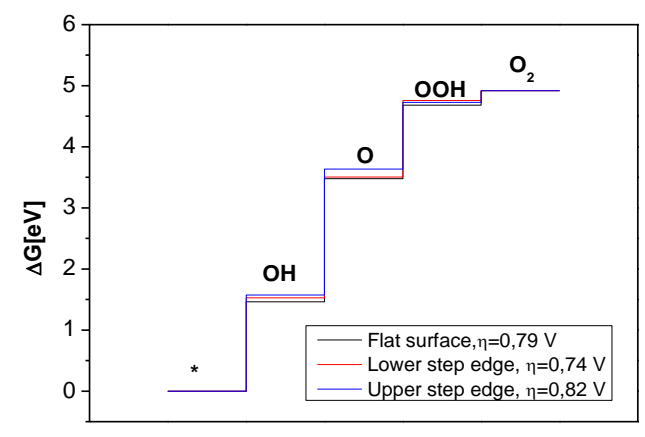

Figure 8. Free energy versus reaction step of the OER on flat surface, lower step edge and upper step edge of (110) hematite surface.

Nguyen et al. investigated water oxidation on defective hematite (0001) surfaces by similar simulations as carried out in this study. ${ }^{31}$ The point defects include $\mathrm{Fe}$ and $\mathrm{O}$ vacancies. They found that $\mathrm{Fe}$ vacancies do not reduce the OER overpotential, whereas $O$ vacancies do lower the overpotential. ${ }^{31}$ More recently, Hellman et al. ${ }^{36}$ found that the effect of oxygen vacancies of reducing the overpotential depends on the surface termination. The authors studied water oxidation on the hydroxyl- and oxygen-terminated hematite (0001) surface. The water oxidation onset potential (1.23 V + overpotential) was determined to be $1.79 \mathrm{~V}$ and $2.09 \mathrm{~V}$ vs. the RHE for the pristine hydroxyland oxygen-terminated hematite, respectively. Hence, without vacancies hydroxyl terminated surface have a lower overpotential towards the OER. The presence of oxygen vacancies, however, resulted in pronounced shifts of the onset potential to $3.09 \mathrm{~V}$ and $1.83 \mathrm{~V}$, respectively. Hence, for the hydroxyl terminated surface, the overpotential increases, while it decreases for the oxygen terminated surface. The authors concluded that electrochemical water oxidation on hematite (0001) is most favorable on the oxygen-terminated surface containing oxygen vacancies. ${ }^{36}$

Here, we study the effect of oxygen vacancies in the hematite (110) surface as this is believed to be the most active surface in PEC water splitting of hematite thin films. ${ }^{47}$ We introduce three different concentrations of oxygen vacancies. The oxygen vacancy systems are constructed based on (110) surfaces as shown in Figure 9. In each system, an oxygen atom (shown in yellow) in a sub-surface layer is removed to create a vacancy. The highest concentration is shown in Figure 9 (a) $(2.52$ vacancies $/ \mathrm{nm}^{2}$ ). By increasing the size of the system to two and four times larger, oxygen vacancy concentration lowers two (1.26 vacancies $\left./ \mathrm{nm}^{2}\right)$ and four $\left(0.63\right.$ vacancies $\left./ \mathrm{nm}^{2}\right)$ times, respectively (Figure $9(\mathrm{~b}, \mathrm{c})$ ). (a)

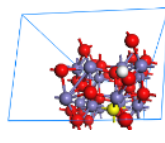

(b)

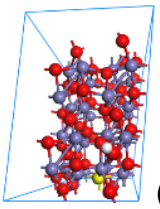

(c)

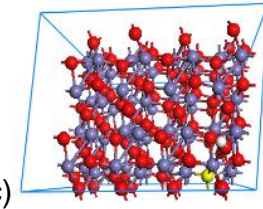

Figure 9. Top view molecular geometries of the hematite (110) surface: The $O$ (in yellow) is removed to form an oxygen sublayer vacancy. The system size increases from (a) to (b) to (c). Thus, the concentration of oxygen vacancies per area decreases. The surface areas are 39.9, 79.8 and $159.6 \AA^{2}$ for (a), (b), and (c) respectively. 
The free energy plots of the three oxygen vacancy systems are shown in Figure 10. Significant differences of the free energies as a function of the vacancy concentration are found. The rate limiting step is again for all geometries the second step (formation of $\mathrm{O})$. The relative stability of $\mathrm{OH}$ and $\mathrm{O}$ for the three oxygen vacancies concentrations are different. Compared to the ideal surface without vacancies, oxygen vacancy concentrations of 2.52 vacancies $/ \mathrm{nm}^{2}$ and 0.63 vacancies $/ \mathrm{nm}^{2}$ stabilize both $\mathrm{OH}$ and $\mathrm{O}$ species. Thus, the overpotential does not change significantly. An oxygen vacancy concentration of 1.26 vacancies $/ \mathrm{nm}^{2}$, however, destabilizes $\mathrm{OH}$, while keeping $\mathrm{O}$ state almost unchanged. This results in a very low overpotential of $0.47 \mathrm{~V}$. This is, to our best knowledge, the lowest overpotential for any $\mathrm{Fe}_{2} \mathrm{O}_{3}$ surface reported by theoretical studies in the literature up to now.

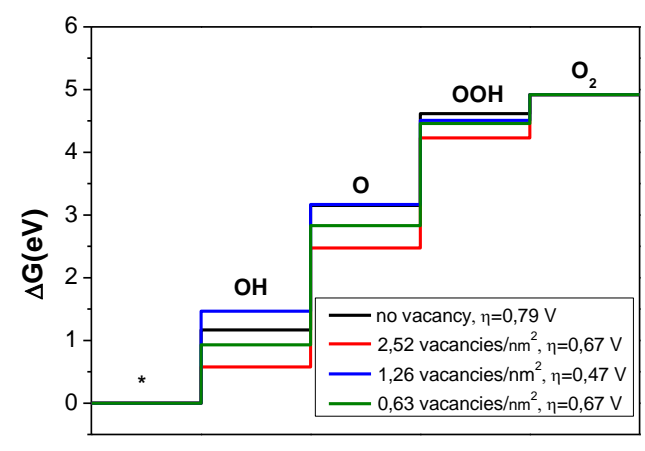

Figure 10. Free energy versus reaction step of the OER as a function of the oxygen vacancy concentration of the (110) surface.

Figure 11 summarizes the overpotentials as a function of oxygen vacancy concentration as calculated in Figure 10 for the hematite (110) surface. The data of Nguyen et al. ${ }^{31}$ and Hellman et al. ${ }^{36}$ for the hematite (0001) surface are included for comparison. The levels of theory are similar for all simulations. From Figure 11, we can see that the (110) surface without vacancies has a lower overpotential than the corresponding (0001) surface and is, hence, more active towards OER. With increasing oxygen vacancy concentration, the overpotential decreases. The same trend was observed by Nguyen et al. ${ }^{31}$ After a minimum overpotential at a concentration of 1.26 vacancies $/ \mathrm{nm}^{2}$, the overpotential increases again. This is also in agreement with the data of Hellman et al. ${ }^{36}$ Therefore, a lowest overpotential of $0.47 \mathrm{~V}$ is obtained with an optimal oxygen vacancy concentration of 1.26 vacancies $/ \mathrm{nm}^{2}$.

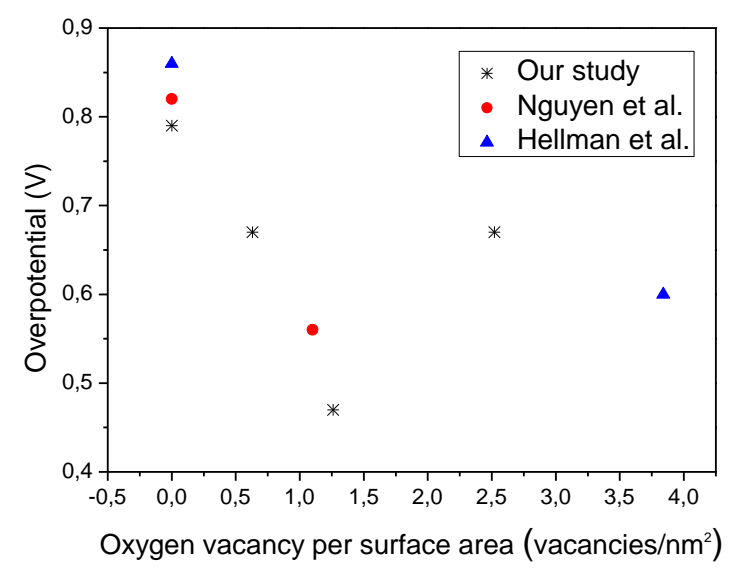

Figure 13. Calculated OER overpotential for the hematite (110) surface as a function of oxygen vacancy concentration. The literature values for the hematite (0001) surface ${ }^{31,36}$ are shown for comparison.

Table 1 summarizes all calculated overpotentials and rate limiting reactions in this study. Although the (110) and (104) orientations were found to have very different experimental PEC activities, ${ }^{47}$ the calculated results show that the difference in electrochemical OER overpotentials is insignificant. The lower step edge is more active than the upper step edge and flat surface due to a lower overpotential. The best strategy to reduce the overpotential and to enhance the activity for OER was found to be creating oxygen vacancies. The lowest overpotential of $0.47 \mathrm{~V}$ (as highlighted in red in Table 1) was found for an oxygen vacancy concentration of 1.26 vacancies $/ \mathrm{nm}^{2}$. It is noteworthy that the rate limiting reaction for the single site mechanism is the deprotonation of $\mathrm{OH}$ to form $\mathrm{O}$, while the rate limiting reaction for the dual site mechanisms is water addition to form $\mathrm{OH}$.

Table 1. Calculated OER overpotential for different hematite surfaces. The lowest overpotential is found for the (110) surfaces with an oxygen vacancy concentration of 1.26 vacancies $/ \mathrm{nm}^{2}$; it is highlighted in red. 


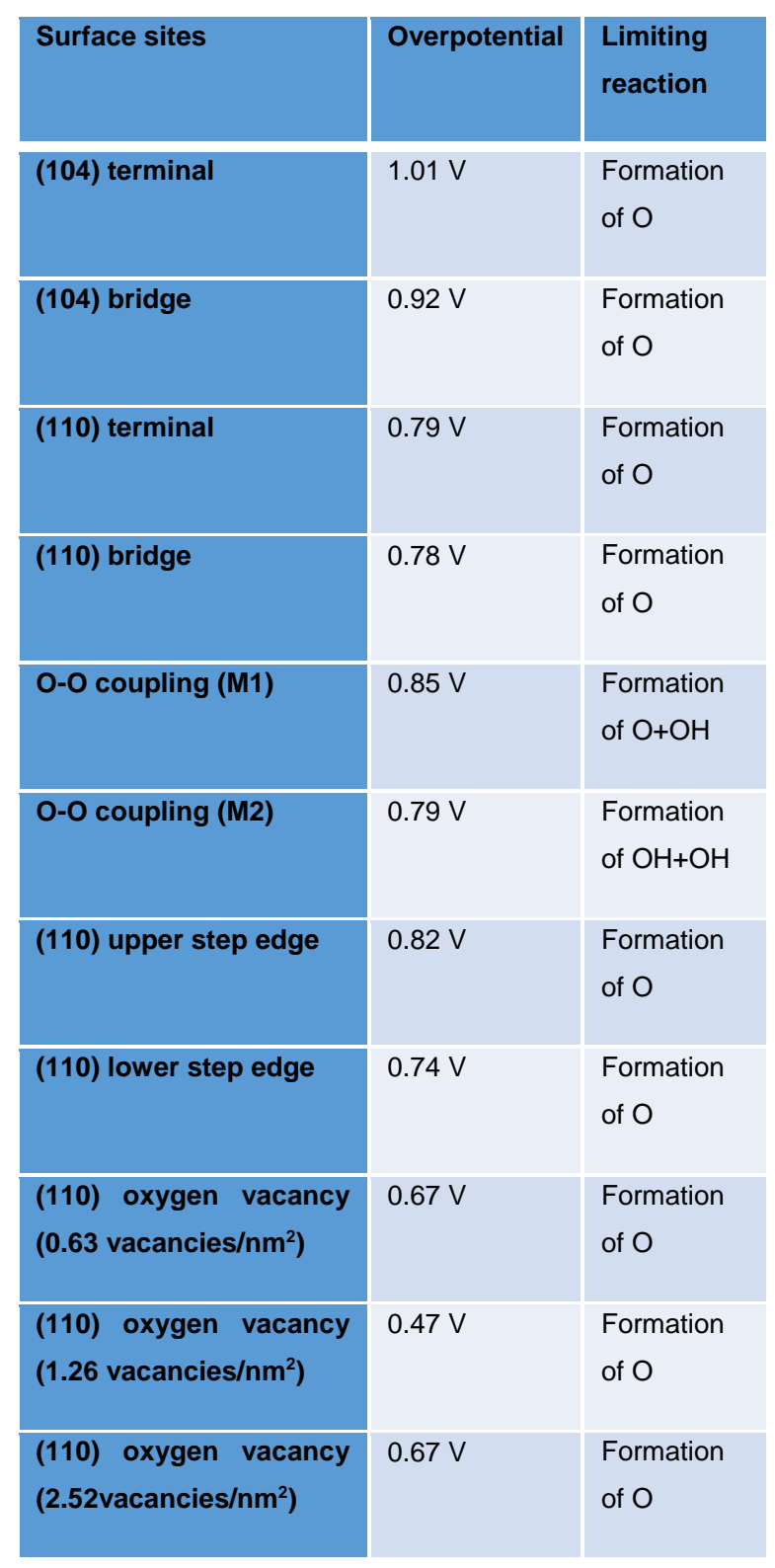

$\mathrm{DFT}+\mathrm{U}$ calculations have been performed to investigate the electrochemical OER overpotential at hematite $\left(\mathrm{Fe}_{2} \mathrm{O}_{3}\right)$ surfaces and to explore the strategies to reduce it. It is found that the oxygen bridge site configuration is slightly more active than the terminal site (lower overpotential). The (110) surface is more active than the (104) surface. Interestingly, the rate limiting reactions are found to be dependent on OER mechanisms. Independent of surface orientation and surface structure, the rate limiting reaction for the single site mechanism is the deprotonation of $\mathrm{OH}$ to form $\mathrm{O}$. For the dual site mechanism (O-O coupling) it is the water addition to form $\mathrm{OH}$. Furthermore, the dual site mechanism at the (110) surface is competing with the single site mechanism. Concerning surface steps, no significant differences in the OER overpotentials are found between flat and stepped surfaces. The by far most efficient way to reduce the overpotential is to control the concentration of oxygen vacancies. An OER overpotential as low as $0.47 \mathrm{~V}$ was obtained for an optimal oxygen vacancy concentration of 1.26 vacancies $/ \mathrm{nm}^{2}$. This is the lowest overpotential ever mentioned by theoretical studies for $\mathrm{Fe}_{2} \mathrm{O}_{3}$. Future work will be carried out to calculate the kinetics of water splitting reactions using a solid-liquid model.

\section{AUTHOR INFORMATION}

Corresponding Author

*Electronic mail: X.Zhang@differ.nl.

^Electronic mail: A.Bieberle@differ.nl.

\section{Notes}

The authors declare no competing financial interest.

\section{ACKNOWLEDGMENTS}

Zhang and Bieberle-Hütter acknowledge the financial support from NWO (FOM program nr. $147{ }^{\prime \prime} \mathrm{CO}_{2}$ neutral fuels") for carrying out this study. Supercomputing facilities of the Dutch national supercomputers SURFsara/Lisa and Cartesius are acknowledged.

\section{REFERENCES}


L. Jia, K. Harbauer, P. Bogdanoff, I. Herrmann-Geppert, A. Ramirez, R. van de Krol and S. Fiechter, Journal of Materials Chemistry A, 2014, 2, 20196-20202.

F. Le Formal, E. Pastor, S. D. Tilley, C. A. Mesa, S. R. Pendlebury, M. Grätzel and J. R. Durrant, J. Am. Chem. Soc., 2015, 137, 66296637.

K. Sivula, F. Le Formal and M. Grätzel, ChemSusChem, 2011, 4, 432-449.

X. Zhang and A. Bieberle-Hütter, ChemSusChem, 2016, 9, 1223-1242.

A. V. Akimov, A. J. Neukirch and O. V. Prezhdo, Chem. Rev., 2013, 113, 4496-4565.

P. Liao and E. A. Carter, Chem. Soc. Rev., 2013, 42, 2401-2422.

A. Valdes, J. Brillet, M. Gratzel, H. Gudmundsdottir, H. A. Hansen, H. Jonsson, P. Klupfel, G.-J. Kroes, F. Le Formal, I. C. Man, R. S. Martins, J. K. Norskov, J. Rossmeisl, K. Sivula, A. Vojvodic and M. Zach, PCCP, 2012, 14, 49-70.

Y. Ping, D. Rocca and G. Galli, Chem. Soc. Rev., 2013, 42, 2437-2469.

Á. Valdés, Z. W. Qu, G. J. Kroes, J. Rossmeisl and J. K. Nørskov, The Journal of Physical Chemistry C, 2008, 112, 9872-9879.

J. Rossmeisl, A. Logadottir and J. K. Nørskov, Chem. Phys., 2005, 319, 178-184.

J. Rossmeisl, Z. W. Qu, H. Zhu, G. J. Kroes and J. K. Nørskov, J. Electroanal. Chem., 2007, 607, 83-98.

Y.-F. Li and Z.-P. Liu, J. Am. Chem. Soc., 2011, 133, 15743-15752.

G.-F. Wei, Y.-H. Fang and Z.-P. Liu, The Journal of Physical Chemistry C, 2012, 116, 12696-12705.

A. Kazaryan, R. van Santen and E. J. Baerends, PCCP, 2015, 17, 20308-20321.

E. Berardo, H.-S. Hu, S. A. Shevlin, S. M. Woodley, K. Kowalski and M. A. Zwijnenburg, Journal of Chemical Theory and Computation, 2014, 10, 1189-1199.

Z. W. Q. J. Rossmeisl, H. Zhu, G. J. Kroes and J. K. Nørskov, J. Electroanal. Chem., 2007, 607, 83-98.

M. C. Toroker and E. A. Carter, Journal of Materials Chemistry A, 2013, 1, 2474-2484.

X. Zhou, E. J. M. Hensen, R. A. van Santen and C. Li, Chemistry - A European Journal, 2014, 20, 6915-6926.

O. Neufeld and M. C. Toroker, The Journal of Physical Chemistry C, 2015, 119, 5836-5847.

B. I. Anders Hellman, Björn Wickman, Henrik Grönbeck, Jonas Baltrusaitis, Surface Science, 2015, 640, 45-49.

M.-T. Nguyen, S. Piccinin, N. Seriani and R. Gebauer, ACS Catalysis, 2015, 5, 715-721.

Y.-H. Fang and Z.-P. Liu, J. Am. Chem. Soc., 2010, 132, 18214-18222.

J. Chen, Y.-F. Li, P. Sit and A. Selloni, J. Am. Chem. Soc., 2013, 135, 18774-18777.

J.-S. Filhol and M. Neurock, Angew. Chem. Int. Ed., 2006, 45, 402-406.

P. Liao, J. A. Keith and E. A. Carter, J. Am. Chem. Soc., 2012, 134, 13296-13309.

A. Hellman, B. Iandolo, B. Wickman, H. Grönbeck and J. Baltrusaitis, Surf. Sci., 2015, 640, 45-49.

P. Liao, M. C. Toroker and E. A. Carter, Nano Lett., 2011, 11, 1775-1781.

M.-T. Nguyen, N. Seriani, S. Piccinin and R. Gebauer, The Journal of Chemical Physics, 2014, 140, 064703.

M.-T. Nguyen, M. Farnesi Camellone and R. Gebauer, The Journal of Chemical Physics, 2015, 143, 034704.

M. C. Toroker, The Journal of Physical Chemistry C, 2014, 118, 23162-23167.

O. Neufeld and M. C. Toroker, PCCP, 2015, 17, 24129-24137.

M.-T. Nguyen, N. Seriani and R. Gebauer, ChemPhysChem, 2014, 15, 3136-3136.

N. Yatom, O. Neufeld and M. Caspary Toroker, The Journal of Physical Chemistry C, 2015, 119, 24789-24795.

K. Ulman, M.-T. Nguyen, N. Seriani and R. Gebauer, The Journal of Chemical Physics, 2016, 144, 094701.

N. Yatom and M. Toroker, Molecules, 2015, 20, 19668.

O. Neufeld, N. Yatom and M. Caspary Toroker, ACS Catalysis, 2015, 5, 7237-7243.

S. Kment, P. Schmuki, Z. Hubicka, L. Machala, R. Kirchgeorg, N. Liu, L. Wang, K. Lee, J. Olejnicek, M. Cada, I. Gregora and R. Zboril, ACS Nano, 2015, 9, 7113-7123.

https://www.vasp.at//).

G. Kresse and D. Joubert, Physical Review B, 1999, 59, 1758-1775.

O. Bengone, M. Alouani, P. Blöchl and J. Hugel, Physical Review B, 2000, 62, 16392-16401.

G. Kresse and J. Furthmüller, Comput. Mater. Sci., 1996, 6, 15-50.

G. Kresse and J. Furthmüller, Physical Review B, 1996, 54, 11169-11186.

J. P. Perdew, K. Burke and M. Ernzerhof, Phys. Rev. Lett., 1996, 77, 3865-3868.

P. E. Blöchl, Physical Review B, 1994, 50, 17953-17979.

R. Frydendal, M. Busch, N. B. Halck, E. A. Paoli, P. Krtil, I. Chorkendorff and J. Rossmeisl, ChemCatChem, 2015, 7, 149-154.

F. Calle-Vallejo, O. A. Díaz-Morales, M. J. Kolb and M. T. M. Koper, ACS Catalysis, 2015, 5, 869-873.

D. K. Kanan, J. A. Keith and E. A. Carter, ChemElectroChem, 2014, 1, 407-415.

J. K. Nørskov, T. Bligaard, A. Logadottir, S. Bahn, L. B. Hansen, M. Bollinger, H. Bengaard, B. Hammer, Z. Sljivancanin, M. Mavrikakis,

Y. Xu, S. Dahl and C. J. H. Jacobsen, J. Catal., 2002, 209 275-278.

R. Nakamura and Y. Nakato, J. Am. Chem. Soc., 2004, 126, 1290-1298.

R. Nakamura, T. Tanaka and Y. Nakato, The Journal of Physical Chemistry B, 2004, 108, 10617-10620.

J. O. Bockris and T. Otagawa, The Journal of Physical Chemistry, 1983, 87, 2960-2971.

A. I. Krasil'shchikov, Zh. Fiz. Khim., 1963, 37, 531-537.

W. O'Grady, C. Iwakura, J. Huang and E. Y. M. W. Brieter, Electrocatalysis, The Electrochemical Society Softbound Proceedings Series, 1974, 286.

R. A. Van Santen, Acc. Chem. Res., 2009, 42, 57-66.

Q. Ge and M. Neurock, J. Am. Chem. Soc., 2004, 126, 1551-1559.

C. P. Plaisance and R. A. van Santen, J. Am. Chem. Soc., 2015, 137, 14660-14672. 